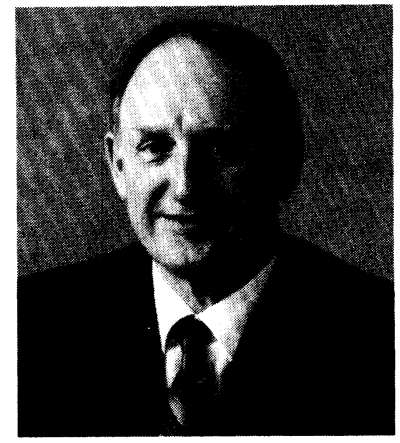

\section{We Are Getting Thicker!}

No, I do not mean your editor, and certainly not the hardworking editorial staff. In response to the flow and acceptance of an increasing number of excellent papers, the Society has increased the page budget for Optical Engineering again. This will allow us to continue to print articles on a timely basis after acceptance. At the moment, it is taking about six or seven months, but we are still trying to reduce that time to our goal of five months.

The October issue of Optical Engineering had 297 technical pages, which brings the total number of technical pages for this year, through October, to 2414. Table 1 shows a comparison of the number of pages published, from January through October, for the last few years.

We had a $41 \%$ increase in the number of pages in 1992 over the previous years. The number of pages last year (1992) and this year (1993) were about the same through the June issue, but then we began adding pages and coming close to 300 pages a month. Thus, by the end of this year Volume 32 will contain approximately 3000 pages. This is a threefold increase since 1988 (Volume 27) and more than a twofold increase since 1991 (Volume 30).

The driving force for this change is the quantity and the quality of the papers submitted to Optical Engineering. Through the end of August of this year we had handled 264 contributed papers and 177 papers associated with special sections. The number of contributed papers has increased by $111 \%$ over the last four years as of the August date. The number of special section papers has increased by a greater percentage than that since there has been tremendous interest in our special sections.

Each month, as your editor, I submit a report to the management of SPIE and to the chair of the Publications Committee. I thought you might like to see the type of data that I prepare. Table 2 shows the activity report for August 1993.
Table 1 Technical pages published from January through October1988 through 1993.

\begin{tabular}{ccc}
\hline Year & $\begin{array}{c}\text { Technical Pages } \\
\text { (10 Months) }\end{array}$ & \% Change \\
\cline { 2 - 2 } 1988 & 922 & - \\
1989 & 1144 & 24.0 \\
1990 & 1318 & 15.2 \\
1991 & 1427 & 8.3 \\
1992 & 2013 & 41.1 \\
1993 & 2414 & 19.9 \\
\hline
\end{tabular}

Table 2 Activity report for August 1993.

\begin{tabular}{|c|c|c|c|}
\hline Papers received during month & 38 & Papers closed & 4 \\
\hline Papers sent to reviewers & 37 & Papers withdrawn & 0 \\
\hline Number of reviewers solicited & 88 & eceived for $\mathrm{fu}$ & \\
\hline Reviews received & 49 & spec & 13 \\
\hline Revised manuscripts received & 19 & $\begin{array}{l}\text { Papers accepted for future } \\
\text { special issues }\end{array}$ & 13 \\
\hline Papers accepted & 16 & Papers for Communications & 1 \\
\hline Papers rejected & 11 & Papers published in August issue & 44 \\
\hline $\begin{array}{l}\text { Papers returned to authors for } \\
\text { revision }\end{array}$ & 14 & $\begin{array}{l}\text { Communications published in } \\
\text { August issue }\end{array}$ & \\
\hline $\begin{array}{l}\text { managing editor } \\
\text { mant }\end{array}$ & 16 & Transferred to other jounals & \\
\hline
\end{tabular}

Rest assured that you, the authors and reviewers, are pressing your editor and the editorial staff in Bellingham to the limit—but that's the way we like it!

The old British proverb says "practice makes perfect." We don't claim to be perfect but we certainly get lots of practice. A more modern American version of that proverb that we might say is "use it or lose it." We are busy using all our faculties and facilities, so I am sure we are not getting thicker-just the journal.

Brian J. Thompson Editor 
December 1993

Magnetospheric Imagery and Atmospheric Remote Sensing, Part 1

Supriya Chakrabarti

Boston University

Center for Space Physics

725 Commonwealth Avenue

Boston, MA 02215

E-mail: supc@bu-ast.bu.edu

617/353-5990 • 617/353-6463 FAX

January 1994

Infrared Technology, Part 1

Marija S. Scholl

Alenka Associates

P.O. Box 27408

Tempe, AZ 85285-7408

E-mail: msscholl@aol.com

602/491-7814

February 1994

Magnetospheric Imagery and

Atmospheric Remote Sensing, Part 2

Supriya Chakrabarti

Boston University

Center for Space Physics

725 Commonwealth Avenue

Boston, MA 02215

E-mail: supc@bu-ast.bu.edu

$617 / 353-5990 \cdot 617 / 353-6463$ FAX

March 1994

Infrared Technology, Part 2

Marija S. Scholl

Alenka Associates

P.O. Box 27408

Tempe, AZ 85285-7408

E-mail: msscholl@aol.com

602/491-7814

April 1994

Optical Pattern Recognition

Joseph L. Horner

Rome Laboratory

EROP

Hanscom AFB, MA 01731-5000

617/377-3841 • 617/377-5041 FAX

Bahram Javidi

University of Connecticut

School of Engineering

Department of Electrical and Systems Engineering

Room 312, U-157

260 Glenbrook Road

Storrs, CT 06269-3157

203/486-4816 • 203/486-3789 FAX

\section{May 1994}

Semiconductor Infrared Detectors

Antoni Rogalski

Military Technical Academy

Institute of Technical Physics

Kaliskiego St. 25

01-489 Warsaw 49, Poland

$4822369109 \cdot 4822362254$ FAX

Optical Interconnects and Packaging

Sing Lee

University of California/San Diego

E\&CE Department

La Jolla, CA 92093-0407

619/534-2413 • 619/534-1225 FAX
June 1994

Optical Science \& Engineering in India

Rajpal S. Sirohi

Indian Institute of Technology

Applied Optics Laboratory

Physics Department

Madras-600 036, India

044-2351365 ext. 221 • 044-2350509 FAX

July 1994

Adaptive Wavelet Transforms

Harold H. Szu

U.S. Navy

Naval Surface Warfare Center

Code R44

10901 New Hampshire Avenue

Silver Springs, MD 20903-5000

301/394-3097 • 301/394-3923 FAX

Manuscripts due Dec. 1, 1993.

August 1994

Digital Image Recovery and Synthesis

Paul S. Idell

Air Force Phillips Lab.

PL/GPOA

390 B Great Road, \#18

Acton, MA 01720

612/377-3663 • 617/377-3661 FAX

Manuscripts due Dec. 1, 1993.

September 1994

Optics in South Africa

Hannes Markusse

ELOPTRO

Institute of Atomic Physics

P.O. Box 869

Kempton Park 1620, South Africa

Maurice W. McDowell

CSIR/Production Technology Div.

Productiontek

P.O. Box 395

Pretoria 0001, South Africa

$27128413418 \cdot 27128412131$ FAX

Manuscripts due Jan. 1, 1994.

October 1994

Optics in Russia

V. Ya. Panchenko

Scientific Research Center for Technological Lasers

Russia Academy of Sciences

B-333, Gubkina, 3

117971 Moscow, Russia

E-mail: ilc@compnet.npimsu.msk.su

(095)135-54-30 • (095)334-02-01 FAX

Manuscripts due March 1, 1994.

November 1994

Micro-Optics

Chandrasekhar Roychoudhuri

University of Connecticut at Storrs

Photonics Research Center

MS-157, Room 312

260 Glenbrook Road

Storrs, CT 06269-3157

203/486-4816 • 203/486-3789 FAX

Manuscripts due April 1, 1994.
December 1994

Optics in Ireland

John Hegarty

C. D. Hussey

University of Dublin

Trinity College

Department of Pure and Applied Physics

Dublin 2, Ireland

+353-1-7022019 • +353-1-711759 FAX

Manuscripts due May 1, 1994.

January 1995

X-Ray/EUV Optics

Richard B. Hoover

NASA Marshall Space Flight Center

ES52 Space Science Center

Huntsville, AL 35812-0001

E-mail: hoover@ssl.msfc.nasa.gov 205/544-7617 • 205/544-5856 FAX

Manuscripts due June 1, 1994.

February 1995

High Heat Flux Optical Engineering

Ali M. Khounsary

Argonne National Laboratory

Advanced Photon Source, APS 362

Argonne, IL 60439

708/252-3384 • 708/252-3222 FAX

Manuscripts due July 1, 1994.

March 1995

Optical Engineering in Ophthalmology

Suganda Jutamulia

Kowa Company, Ltd

Silicon Valley Office

100 Homeland Court, Suite 302

San Jose, CA 95112

408/441-9300 • 408/441-0537 FAX

Toshimitsu Asakura

Hokkaido University

Research Institute for Electronic Science

Sapporo, 060 Japan

81-11-716-2111 • 81-11-758-3173 FAX

Manuscripts due Aug. 1, 1994.

April 1995

Optics in the Ukraine

Oleg V. Angelsky

Chernovtsy University

Department of Correlation Optics

2 Kosyubinsky Street

274012 Chernovtsy

Ukraine

(03722) 44730 • (03722) 41314 FAX

Manuscripts due Aug. 15, 1994

July 1995

Optics in Switzerland

P. K. Rastogi

Swiss Federal Institute of Technology-Lausanne

Laboratory of Stress Analysis

CH-1015 Lausanne

Switzerland

E-mail: rastogi@elgc.epfl.ch

(021) 6932445 •(021) 6934748 FAX

Manuscripts due Nov. 15, 1994 